\title{
Incidence of injury and illness in South African professional male football players: A prospective cohort study
}

Helen Bayne ${ }^{1,2^{*}}$, Martin Schwellnus ${ }^{1}$, Dina Janse Van Rensburg ${ }^{1}$, Jhano Botha ${ }^{3}$, Lervasen Pillay ${ }^{1}$

${ }^{1}$ Institute for Sport, Exercise Medicine and Lifestyle Research \& Section Sports Medicine, Faculty of Health Sciences, University of Pretoria, South Africa

${ }^{2}$ High Performance Centre, University of Pretoria, South Africa

${ }^{3}$ University of Pretoria Football Club, South Africa

*Corresponding Author: Helen Bayne

Email Address: $\quad$ helen.bayne@hpc.co.za

Postal Address: $\quad$ High Performance Centre, LC de Villiers Sports Grounds

Burnett Street

Hatfield 0083

Pretoria, South Africa 


\begin{abstract}
BACKGROUND: Medical illnesses and sports-related injuries both have an effect on athlete health and performance. Epidemiology of injury and illness has been extensively researched during international football tournaments and the European football season. Reports on injury location and severity differ across geographical regions, and there is limited information on injury epidemiology in African football leagues. No studies have investigated the illness burden in football in Africa.
\end{abstract}

METHODS: This was a prospective cohort study involving two football teams over the 10month duration of the 2015/16 Premier Soccer League in South Africa. Team medical staff recorded daily football exposure, illness and injuries. Team-based match and training exposure was calculated and used to determine injury and illness incidence and burden over the football season.

RESULTS: Overall injury incidence was 2.2 / $1000 \mathrm{~h}$, with match injury incidence of 24.8 / $1000 \mathrm{~h}$ and training injury incidence of $0.9 / 1000 \mathrm{~h}$. Time loss injuries accounted for 33 of the 44 injuries recorded. The most common time loss injury location was the knee (14 injuries, 42\%). There were 7 minimal, 4 mild, 12 moderate and 10 severe injuries. Sprain/ligament injury (8 injuries) was the most common type, followed by meniscus/cartilage injury (7 injuries). Eleven illnesses were reported during the season, with an incidence of 0.7 / 1000 player days, and most were minimal in severity (8/11). The illness burden was 1.7 / 1000 player days. The respiratory (46\%) and gastrointestinal (36\%) systems were most commonly affected.

CONCLUSIONS: The incidence of injury was comparable with data reported internationally and mirrors the increased risk of injury during matches versus training. The nature of injury differed in that the knee was more frequently affected than the ankle or thigh, joint injuries were more common than muscle injuries, and there was a larger proportion of severe injuries. The illness burden was very low.

Keywords: Football; Sports injury; Illness; Epidemiology 


\section{Introduction}

Injury prevention and the protection of athlete health is of vital importance on a global level to promote safety in sport, for sports teams to maximise their chance of success, and for individuals to enable optimal performance and ensure long-term health. ${ }^{1,2}$ Sports-related injuries and medical illnesses both have an effect on athlete health and performance.

Epidemiology of injury and illness in football players has been extensively researched during international tournaments (including FIFA World Cups, FIFA Confederations Cups, and the Olympic Games). ${ }^{3-5}$ However, these tournament-based studies do not reflect the overall injury and illness burden, as they do not consider the total football season. Current knowledge on football injury epidemiology during a season is largely based on the Union of European Football Associations (UEFA) injury study, a prospective cohort study involving numerous teams participating in the UEFA league since $2001 .^{6}$ They showed consistent data over seven football seasons, with an overall injury incidence of $8 / 1000$ hours. Match injuries had a higher incidence (28 / 1000 hours) than training injuries (4 / 1000 hours). These data have shown the incidence of injury in elite male football to be high, with the most common type of injury being a hamstring muscle strain (12\% of all injuries). Studies on elite male football players in other regions of the world have been conducted on a smaller scale. In the United States, a single season study involving ten teams participating in Major League Soccer reported similar overall injury incidence rates with higher incidence in matches than training. ${ }^{7}$ However, in this study, knee and ankle joint injuries were more common than hamstring strains. In a single season of the Hong Kong professional football league, lower overall injury rates but higher match injury rates have been reported, with the ankle being the most common injury location. ${ }^{8}$

These regional variations suggest that local data is required to guide injury prevention research and intervention programmes that address the main injury concerns in a particular region or league. In Africa, a prospective cohort study in Nigerian players reported injuries sustained during matches and training but only recorded match exposure, so overall incidence could not be calculated. ${ }^{9}$ A South African study involving one professional team over a single football season reported a much higher match injury incidence ( $89 / 1000$ hours) than the European data, with the thigh and ankle most frequently injured. ${ }^{10}$

In an attempt to address overall athlete health, there is a growing research interest in medical illness in football players. A three-year prospective study involving an elite French club team 
reported that each player suffered from 2.5 illness complaints per season, but only $20 \%$ of these episodes resulted in players being unavailable for training or matches. ${ }^{11}$ Over a fouryear period of the UEFA study, the illness incidence was 1.5 / 1000 player days, meaning that players sustained 0.4 illnesses per season, on average. Both studies found that respiratory tract and gastrointestinal illnesses were most frequent, and that the illness burden was relatively low (less than $1 \%$ of training or match days lost). ${ }^{12}$

There is a need to expand on these South African injury data and also to consider other aspects of player health, namely medical illnesses, in order to better understand the medical needs of elite male football players in this region. Therefore, the aim of this study was to investigate the incidence and nature of medical illnesses and football-related injuries in elite male football players in South Africa.

\section{Materials and methods}

This was a prospective cohort study over the 10-month duration of the 2015/16 Premier Soccer League in South Africa.

The study was conducted by the University of Pretoria in cooperation with two football teams participating in the Premier Soccer League. Before the start of the season, research ethics approval was obtained from the Faculty of Health Sciences Research Ethics Committee (REF 205/2015). Detailed information about all components of the study was provided to the medical staff of the participating teams, and all players in each of the two teams $(n=56)$ were approached through the respective medical staff to participate in the study. A comprehensive subject information sheet was provided to each player, explaining the details of the study as well as all the potential risks and benefits. Written informed consent was obtained from all players.

The type of day (rest, training or match) and number of training hours were recorded daily by the team medical staff and captured electronically. Team-based match and training hours were calculated from these data, according to the consensus statement of Fuller and colleagues. ${ }^{13}$

All injuries that resulted in a player receiving medical attention (medical attention injuries) and injuries that resulted in a player being unable to take a full part in future football training 
or match play (time loss injury) were documented and classified by location and type. ${ }^{13}$ Team medical staff also recorded the circumstances in which the injury occurred (contact, noncontact, or foul play), training or match days lost due to injury, and a diagnosis using the Orchard Sports Injury Classification System (OSICS) version 10.1. ${ }^{14}$ The incidence of injury was calculated as the number of injuries per 1000 player hours, for training and match injuries. The injury burden was calculated as the number of injury days lost per 1000 player hours, thus accounting for both frequency and severity of injuries. ${ }^{15}$

Medical illnesses, defined as "any non-trauma related symptom or sign presenting in a player that required medical attention", ${ }^{16}$ were assessed and documented by team medical staff. The information recorded included presenting signs or symptoms, clinical diagnosis (using OSICS 10.1), and predicted number of training or match days that would be lost due to illness. The incidence of illness was calculated as the number of illnesses per 1000 player days. Illness burden was calculated as the number of days lost due to illness per 1000 player days. ${ }^{12}$

Injury and illness severity was based on the number of days from the date of presentation until return to play and categorised as follows: slight (0 days), minimal (1-3 days), mild (4-7 days), moderate (8-28 days) and severe (>28 days). ${ }^{13}$

\section{Results}

In total, 16920 player days and 20361 hours of football exposure were registered (including 1089 match hours). The mean football exposure per player during the surveillance period was 364 hours.

\section{Injury incidence}

Overall injury incidence (medical attention and time loss injuries combined) was $2.2 / 1000 \mathrm{~h}$, with match injury incidence of $24.8 / 1000 \mathrm{~h}$ and training injury incidence of $0.9 / 1000 \mathrm{~h}$. Considering time loss injuries alone, overall incidence was $1.6 / 1000 \mathrm{~h}$, match incidence was $16.5 / 1000 \mathrm{~h}$, and training incidence was $0.8 / 1000 \mathrm{~h}$. Medical attention injuries only occurred during matches, with a match incidence of $8.3 / 1000 \mathrm{~h}$.

Time loss injuries accounted for 33 of the 44 injuries recorded (Table 1). The injury burden was 63.5 days lost / $1000 \mathrm{~h}$. The most common time loss injury location was the knee (14 
injuries, 42\%). There were 7 minimal, 4 mild, 12 moderate and 10 severe injuries. Sprain/ligament injury (8 injuries) was the most common type, followed by meniscus/cartilage injury (7 injuries). These also accounted for the largest proportion of lost playing time $-36 \%$ and $28 \%$ respectively (Table 2 ). Of the time loss injuries, 11 occurred in contact situations, 19 in non-contact situations and 3 involved foul play.

Table I. Time loss injury pattern by location and severity

\begin{tabular}{lcccccc}
\hline & $\begin{array}{c}\text { Number of } \\
\text { injuries }\end{array}$ & Minimal & Mild & Moderate & Severe & $\begin{array}{c}\text { Total Days } \\
\text { Lost }\end{array}$ \\
\hline Knee & $14(42)$ & 2 & 2 & 4 & 6 & $902(74)$ \\
Ankle & $5(15)$ & 2 & & 1 & 2 & $119(10)$ \\
Lower leg & $4(12)$ & & 1 & 2 & 1 & $89(7)$ \\
Hip/groin & $3(9)$ & & & 2 & 1 & $66(5)$ \\
Thigh & $2(6)$ & 1 & & 1 & & $11(1)$ \\
Head/face & $2(6)$ & 1 & & 1 & & $13(1)$ \\
Shoulder/clavicle & $1(3)$ & & & 1 & & $15(1)$ \\
Sternum/ribs/upper back & $1(3)$ & 1 & & & & 2 \\
Foot/toe & $1(3)$ & & 1 & & & 7 \\
Total & $33(100)$ & 7 & 4 & 12 & 10 & 1224 \\
\hline Values in brackets show
\end{tabular}

Values in brackets show percentage of total

Table II. Time loss injury pattern by type and severity

\begin{tabular}{lcccccc}
\hline & $\begin{array}{c}\text { Number of } \\
\text { injuries }\end{array}$ & Minimal & Mild & Moderate & Severe & Total Days \\
Lost
\end{tabular}


Eleven medical attention injuries were reported and consisted mainly of contusions/bruises $(55 \%)$ and lacerations (27\%). The head (27\%), hand/finger (18\%) and ankle (18\%) were most frequently involved.

\section{Illness incidence}

Eleven illnesses were reported during the season, with an incidence of 0.7 / 1000 player days, and most were minimal in severity (8/11). The illness burden was 1.7 / 1000 player days. The respiratory (46\%) and gastrointestinal (36\%) systems were most commonly affected (Table 3). One severe illness affected the cardiovascular system, forcing the player to end his season. This episode was excluded from the illness burden calculation to avoid skewing the "days lost" data.

Table III. Illness pattern by severity

\begin{tabular}{lccccc}
\hline & $\begin{array}{c}\text { Number of } \\
\text { illnesses }\end{array}$ & Minimal & Mild & Moderate & Severe \\
\hline Respiratory & $5(46)$ & 4 & 1 & & \\
Gastrointestinal & $4(36)$ & 4 & & & \\
Cardiovascular & $1(9)$ & & & & 1 \\
Other & $1(9)$ & & & 1 & \\
Total & $11(100)$ & 8 & 1 & 1 & 1 \\
\hline Values in brackets show percentage of total & & & &
\end{tabular}

\section{Discussion}

This is the largest prospective cohort study to be conducted on football-related injury incidence in Africa, and the first to include illness over the duration of a full football season. The main findings were (1) that, over a 10-month surveillance period, the overall injury incidence was 2.2 / 1000 player hours, (2) that match injury incidence was substantially higher than training injury incidence, (3) that the knee was the most commonly injured location and also accounted for the majority of lost playing time, and (4) that the incidence (0.7 / 1000 player days) and burden (1.7 / 1000 player days) of illness was low.

Previous studies using similar methodology in Europe, the United States, Asia and South Africa have demonstrated a training injury incidence of $2.9-6.6 / 1000 \mathrm{~h}$, and a match injury incidence of $14.5-88.9 / 1000$ h..$^{6-8,10,17}$ The training $(0.9 / 1000 \mathrm{~h})$ and match $(24.8 / 1000 \mathrm{~h})$ 
incidence in the present study is at the lower end of these ranges and follows the same trend for greatly increased injury risk during football matches compared to training.

Thirty percent of all time loss injuries were classified as severe (requiring more than 28 days to recover). This represents a larger proportion of severe injuries than the studies conducted in other regions $(7-25 \%){ }^{6-8,17}$ Another South African based study reported 56\% of injuries sustained were severe. ${ }^{10}$ The time to return to play is affected by the extent of the pathology as well as the rehabilitation and return to sport decisions made by the medical staff, team and player. Severe injuries may have long-term implications for players and the resultant injury burden impacts on team performance. Future research should therefore aim to investigate the nature of severe injuries and their management, and whether factors unique to the South African environment are related to increased injury severity and associated time loss.

The knee was by far the most frequently recorded injury location, with $42 \%$ of time loss injuries involving this joint, in contrast with other studies that have reported thigh muscle and ankle joint injuries to be more common. ${ }^{7,8,10,18}$ These knee injuries also tended to be more serious, with 6 injuries requiring more than 28 days before return to play. Almost threequarters of all player days lost were the result of knee injuries. Follow up studies should examine whether this is consistent amongst other clubs and across seasons and investigate the mechanism of injury.

Joint (sprain/ligament and meniscus/cartilage) injuries were most common, followed by tendon injuries. Together, these accounted for $87 \%$ of lost playing time. Interestingly, the incidence of muscle strain injury was low. This may reflect the nature of the game played in South Africa compared to other parts of the world and suggests that fewer high speed running activities and more change of direction activities may be present in the South African game.

Previous research has described a low incidence and burden of illness in professional football, with the respiratory and gastrointestinal systems most commonly affected. ${ }^{12}$ The findings of the present study support this, with even fewer illness episodes than comparable studies. In the UEFA study, an illness burden of 7 lost days per 1000 player days, although low, is more than 4 times greater than the current study. Only $20 \%$ of players suffered from an illness that forced them to miss at least one day, compared with $36 \%$ over a three-year period in an elite French club team. ${ }^{11}$ Possible reasons for this lower incidence of illness may be underreporting by players to their team doctor, seeking medical support outside of the team medical staff, or prophylactic interventions. 
This study recorded football exposure data on a group basis, and the team match and training exposure was calculated accordingly. This approach does not take into account exposure time lost when a player does not complete a full training session. In order to investigate specific risk factors for certain injuries, individual exposure data would be required. ${ }^{13}$

The study design, methodology and injury definitions utilised favour acute injuries and may underrepresent overuse or gradual onset injuries. ${ }^{19}$ Using the current methodology, injuries were recorded when the player presented to medical staff and the impact of the injury was denoted as days until return to play. In the case of overuse injuries, the pathological process and development of symptoms often begins well before the player seeks medical attention. Athletes often continue to train with overuse injuries in the presence of pain, but this may affect their performance and subsequent injury risk. An individualised approach, with regular player feedback on symptoms and function, should be employed in order to capture overuse injuries as well as acute injuries and illnesses, in order to achieve a more comprehensive view of player health. ${ }^{20}$

\section{Conclusions}

In summary, this prospective cohort study in two professional football teams who participated in a 10-month season in South African Premier Soccer League showed that the illness burden is very low. The incidence of injury is comparable with data reported internationally and mirrors the increased risk of injury during matches versus training. The nature of injury differs in that the knee was more frequently affected than the ankle or thigh, joint injuries were more common than muscle injuries, and there was a larger proportion of severe injuries.

\section{Acknowledgments}

The authors would like to thank the football clubs who participated in this study. Funding for the project has been provided by the FIFA Legacy Trust. 


\section{REFERENCES}

1. Dvorak J, Junge A. Twenty years of the FIFA Medical Assessment and Research Centre: from 'Medicine for Football' to 'Football for Health'. Br J Sports Med. 2015;49(9):561-3.

2. Steffen K, Soligard T, Engebretsen L. The IOC's endeavour to protect the health of the athlete continues. Br J Sports Med. 2011;45(7):551-2.

3. Dvorak J, Junge A, Derman W, Schwellnus M. Injuries and illnesses of football players during the 2010 FIFA World Cup. Br J Sports Med. 2011;45(8):626-30.

4. Junge A, Dvorak J. Injury surveillance in the World Football Tournaments 1998-2012. Br J Sports Med. 2013;47(12):782-8.

5. Theron N, Schwellnus M, Derman W, Dvorak J. Illness and injuries in elite football players--a prospective cohort study during the FIFA Confederations Cup 2009. Clin J Sport Med. 2013;23(5):379-83.

6. Ekstrand J, Hagglund M, Walden M. Injury incidence and injury patterns in professional football: the UEFA injury study. Br J Sports Med. 2011;45(7):553-8.

7. Morgan BE, Oberlander MA. An examination of injuries in major league soccer. The inaugural season. Am J Sports Med. 2001;29(4):426-30.

8. Lee JW-Y, Mok K-M, Chan HC-K, Yung PS-H, Chan K-M. A prospective epidemiological study of injury incidence and injury patterns in a Hong Kong male professional football league during the competitive season. Asia-Pacific Journal of Sports Medicine, Arthroscopy, Rehabilitation and Technology. 2014;1(4):119-25.

9. Ani U, Ibikunle P, Akosile C, Useh U. The UEFA model in identification of types, severity and mechanism of injuries among professional footballers in the Nigerian Premier League. S Afr J Sports Med. 2015;27(1):12-5.

10. Calligeris T, Burgess T, Lambert M. The incidence of injuries and exposure time of professional football club players in the Premier Soccer League during football season. S Afr J Sports Med. 2015;27(1):16-9.

11. Orhant E, Carling C, Cox A. A three-year prospective study of illness in professional soccer players. Res Sports Med. 2010;18(3):199-204.

12. Bjorneboe J, Kristenson K, Walden M, Bengstonn H, Ekstrand J, Hagglund M, et al. Role of illness in male professional football: not a major contributor to time loss. Br J Sports Med. 2016;50(11):699-702. 
13. Fuller CW, Ekstrand J, Junge A, Andersen TE, Bahr R, Dvorak J, et al. Consensus statement on injury definitions and data collection procedures in studies of football (soccer) injuries. Br J Sports Med. 2006;40(3):193-201.

14. Rae K, Orchard J. The Orchard Sports Injury Classification System (OSICS) version 10. Clinical Journal of Sports Medicine. 2007;17(3):201-4.

15. Hägglund $\mathrm{M}$, Waldén $\mathrm{M}$, Magnusson $\mathrm{H}$, Kristenson $\mathrm{K}$, Bengtsson $\mathrm{H}$, Ekstrand $\mathrm{J}$. Injuries affect team performance negatively in professional football: an 11-year followup of the UEFA Champions League injury study. Br J Sports Med. 2013.

16. Schwellnus M, Derman W, Page T, Lambert M, Readhead C, Roberts C, et al. Illness during the 2010 Super 14 rugby union tournament - a prospective study involving 22 676 player days. Br J Sports Med. 2012;46:499-504.

17. Eirale C, Farooq A, Smiley F, Tol J, Chalabi H. Epidemiology of football injuries in Asia: A prospective study in Qatar. J Sci Med Sport. 2013;16(2):113-7.

18. Ekstrand J, Hagglund M, Walden M. Epidemiology of muscle injuries in professional football (soccer). The American Journal of Sports Medicine. 2011;39(6).

19. Bahr R. No injuries, but plenty of pain? On the methodology for recording overuse symptoms in sports. Br J Sports Med. 2009;43(13):966-72.

20. Clarsen B, Rønsen O, Myklebust G, Flørenes TW, Bahr R. The Oslo Sports Trauma Research Center questionnaire on health problems: a new approach to prospective monitoring of illness and injury in elite athletes. Br J Sports Med. 2014;48(9):754-60. 